九州大学学術情報リポジトリ

Kyushu University Institutional Repository

\title{
OPTIMUM DESIGNS FOR POLYNOMIAL APPROXIMATION
}

Tasaka, Masao

https://doi.org/10.5109/13029

出版情報：統計数理研究. 13 (1/2)，pp.25-39，1968-03. Research Association of Statistical Sciences

バージョン :

権利関係 : 


\title{
OPTIMUM DESIGNS FOR POLYNOMIAL APPROXIMATION
}

By

\author{
Masao TASAKA \\ (Received November 10,1967)
}

\section{$\S 0$. Introduction.}

In the regression analysis the problem of finding optimum design that minimizes a variance error due to an inadequacy of fitted polynomial has been investigated by many authors under the assumption that the expectation of fitted polynomial is always unbiased on a given interval of the independent variable. And these optimum designs have been established by Smith [17], Chernoff [3], Ehrenfeld [6], Guest [8], Hoel [9], Kiefer and Wolfowitz [11] and others.

Kitagawa [14] has proposed a formulation related to a common problem between the numerical analysis and mathematical statistics. Namely, he discussed the polynomial approximation by Lagrange's interpolation formula from the standpoints of stochastically approximative analysis. The similar problem in some simple cases was studied by David and Arrens [4]: they treated only the case of fitting a straight line when the true function is roughly linear and may contain a quadratic component. Furthermore, Box and Draper [1], [2] have investigated the particular cases where the fitted polynomial in the continuous variables is of the first or the second degree and where true regression function is a polynomial of the second or the third degree. And those optimum designs were selected so as to minimize the expected mean square error.

The purpose of this paper is to explain the relationship among some optimum designs which satisfy the same criteria of optimality with those described by Kitagawa [14] and Hoel [9] and others. The optimum designs for the polynomial interpolation which are well known in the numerical analysis are introduced in section 2. An optimum design derived by Guest [8] and Hoel [9] is introduced in section 3 . And the extension of Hoel's result is completely given there. Furthermore, it is shown that Guest and Hoel's optimum design satisfies another criterion of optimality. In section 4 , the problem which was given by David and Arrens [4] is extended according to our formulation defined in section 1. Finally, the minimum efficiency of the designs obtained in section 2 and 3 will be investigated in section 5 .

\section{$\S 1$. Preliminaries and definitions.}

Let $f(x)$ be a true regression function on the given closed interval $[-1,1]$ of the independent (continuous) variable $x$. And it is assumed that $f(x)$ is $p$ times differen- 
tiable and has the continuous derivative $f^{(p)}(x)$. Let $y_{i j}\left(i=1,2, \cdots, p ; j=1,2, \cdots, n_{i}\right)$ be the $n\left(=\sum_{i=1}^{p} n_{i}\right)$ dependent variables observed on the $p$ distinct points $x_{i}(i=1,2$, $\cdots, p)$, which we shall call the supporting points, and it is assumed that $y_{i j}$ s are independently distributed with mean $f\left(x_{i}\right)$ and common variance $\sigma^{2}$. The design of experiment in the regression problem is determined by an assignment of $\left(x_{1}, \cdots, x_{p}\right.$; $\left.n_{1}, \cdots, n_{p}\right)$, which we shall call a design $d$. And let $\Delta$ denote the class of all possible such designs for fixed $n$ and $p$.

Usually, the polynomial approximation is used for estimating an unknown function $f(x)$ from the experimental results which are obtained by the given design. Throughout this paper we restrict to the problem of fitting regression curve by using Lagrange's interpolation formula, and then under the design $d$ the estimated equation, $\hat{f}_{p-1}(x ; d)$, of true regression function $f(x)$ is given by

where

$$
\hat{f}_{p-1}(x ; d)=\sum_{i=1}^{p} L_{i}(x) \bar{y}_{i},
$$

$$
\left.\begin{array}{l}
L_{i}(x)=F(x) /\left(x-x_{i}\right) F^{\prime}\left(x_{i}\right), \\
F(x)=\prod_{i=1}^{p}\left(x-x_{i}\right),
\end{array}\right\}
$$

and

$$
\bar{y}_{i}=\sum_{j=1}^{n_{i}} y_{i j} / n_{i} .
$$

In this case the expectation of the bias

is given by

$$
\rho(x ; d)=f(x)-\hat{f}_{p-1}(x ; d)
$$

$$
E[\rho(x ; d)]=F(x) \frac{f^{(p)}(\xi)}{p !},
$$

where $-1<\xi<1$.

It is easily shown that

$$
E\left[\rho^{2}(x ; d)\right]=E^{2}[\rho(x ; d)]+\sigma^{2} \sum_{i=1}^{p} \frac{L_{i}^{2}(x)}{n_{i}} .
$$

We wish to determine the optimum design in $\Delta$ so as to minimize $E\left[\rho^{2}(x ; d)\right]$ in some sense. In considering such situation, the case, where $\sigma^{2}=0$ is assumed, has been mainly investigated in the field of numerical analysis. On the other hand, the case, where $E[\rho(x ; d)]=0$ is assumed, belongs to the category of mathematical statistics. When these assumptions are satisfied, we say that the former is the deterministic case and the latter is the unbiased stochastic case respectively. In general the case which remains unexplained on both $f(x)$ and $\sigma^{2}$ is said to be the stochastic case.

Now, $E\left[\rho^{2}(x ; d)\right]$ depends upon the independent variable $x$, so that we have to define the criterion of optimality on such design so as to minimize some functional of $E\left[\rho^{2}(x ; d)\right]$.

In order to do this we first take up two quantities which should be minimized among the class $\Delta$ of all possible designs as follows: the expected mean square error is represented by 


$$
V\left[d, f^{(p)}, \sigma, \alpha\right]=\int_{-1}^{1} E\left[\rho^{2}(x ; d)\right] d \alpha(x),
$$

where $\alpha(x)$ is nondecreasing function in $[-1,1]$ and not constant, and the maximum expected squared error is represented by

$$
V\left[d, f^{p}, \sigma\right]=\max _{-1 \leqq x \leqq 1} E\left[\rho^{2}(x ; d)\right]
$$

Thus we define two optimum designs as follows.

Definition 1. A design $d^{*}\left(f^{(p)}, \sigma, \alpha\right)$ in $\Delta$ is said to be $I\left(f^{(p)}, \sigma, \alpha\right)$-optimum if And for any $d \in \mathcal{L}$,

$$
V\left[d^{*}\left(f^{(p)}, \sigma, \alpha\right), f^{(p)}, \sigma, \alpha\right]=\min _{d \in \boldsymbol{\Delta}} V\left[d, f^{(p)}, \sigma, \alpha\right] .
$$

$$
e\left[d \mid f^{(p)}, \sigma, \alpha\right]=V\left[d^{*}\left(f^{(p)}, \sigma, \alpha\right), f^{(p)}, \sigma, \alpha\right] / V\left[d, f^{(p)}, \sigma, \alpha\right]
$$

is said the efficiency of $d$.

Definition 2. A design $d^{*}\left(f^{(p)}, \sigma\right)$ in $\Delta$ is said to be $G\left(f^{(p)}, \sigma\right)$-optimum if

And for any $d \in \Delta$,

$$
V\left[d^{*}\left(f^{(p)}, \sigma\right), f^{(p)}, \sigma\right]=\min _{d=\Delta} V\left[d, f^{(p)}, \sigma\right] .
$$

$$
e\left[d \mid f^{(p)}, \sigma\right]=V\left[d^{*}\left(f^{(p)}, \sigma\right), f^{(p)}, \sigma\right] / V\left[d, f^{(p)}, \sigma\right]
$$

is said the efficiency of $d$.

\section{$\S 2$. Deterministic optimum designs.}

The parameter $\xi$ in (1.4) depends, in general, not only upon the $p$ supporting points $x_{1}, x_{2}, \cdots, x_{p}$ but also upon the variable $x$ and the nature of true function $f(x)$. In the deterministic case, therefore, it is difficult to seek the designs which satisfy $I\left(f^{(p)}, 0, \alpha\right)$ and $G\left(f^{(p)}, 0\right)$-optimality. Accordingly the approximative optimal spacing which minimizes

$$
\int_{-1}^{1} F(x ; d) d \alpha(x) \quad \text { or } \quad \max _{-1 \leqq x \leqq 1}|F(x ; d)|
$$

has been studied in the numerical analysis. This solution is exactly optimum if

$$
f^{(p)}(x)=c_{p}(=\text { constant })
$$

are true. For these cases, we will rewrite two well-known results in the numerical analysis according to our notation as follows :

" $I\left(c_{p}, 0, \alpha\right)$-optimum design $d^{*}\left(c_{p}, 0, \alpha\right)$ is the design in which the p supporting points consist of the zeros of orthogonal polynomial $\phi_{p}(x)$ of degree $p$ associated with the distribution $d \alpha(x)$ on $[-1,1]$ and in which $n_{1}, n_{2}, \cdots, n_{p}$ are a set of positive integers such that $\sum_{i=1}^{p} n_{i}=n . "$

" $G\left(c_{p}, 0\right)$-optimum design, that is $d^{*}\left(c_{p}, 0\right)$, coincides with $d^{*}\left(c_{p}, 0,\left(1-x^{2}\right)^{-{ }^{1}}{ }^{1}\right) . "$

\section{§3. Unbiased stochastic optimum designs.}

Let $n_{1}, n_{2}, \cdots, n_{p}, a$ and $\zeta$ be the positive integers, and let the relation 
hold true.

$$
\sum_{i=1}^{p} n_{i}=a p+\zeta, \quad(0 \leqq \zeta<p)
$$

The following result was given by Guest [8]: "When $\zeta=0, G(0, \sigma)$-optimum design $d^{*}(0, \sigma)$ is the design in which p supporting points consist of the zeros of $\int_{-1}^{1} P_{p-1}(x) d x$ and $n_{1}=\cdots=n_{p}=a$, where $P_{p-1}(x)$ is Legendre's polynomial of degree $p-1 . "$

Hoel [9] investigated the $D$-optimum design which minimizes the generalized variance of the best linear estimators of the coefficients of polynomial regression $f(x)$ $=\sum_{i=0}^{p-1} c_{i} x^{i}$, and noted the fact which is equivalent to the following:

"When $\zeta=0$, D-optimum design in the polynomial regression coincides with Guest's $G(0, \sigma)$-optimum design, that is, $d^{*}(0, \sigma)$."

In such design it is required that $n / p$ is an integer a, i. e. $\zeta=0$. For the sake of generalization, we shall first provide the following lemma.

LEMMA. A set of $p$ positive integers $\left(n_{1}, n_{2}, \cdots, n_{p}\right)$ which maximize $\prod_{i=1}^{p} n_{i}$ under the restriction $\sum_{i=1}^{p} n_{i}=n$ (=constant) satisfies the condition

$$
\left|n_{\mu}-n_{\nu}\right| \leqq 1 \quad \text { for all } \mu, \nu(=1,2, \cdots, p) .
$$

Proof. When $p=2$, this lemma is trivial. Now suppose that $\prod_{i=1}^{p} n_{i}$ attaines to the maximum value by $n_{1}^{0}, n_{2}^{0}, \cdots, n_{p}^{0}$. For any pair $n_{\mu}^{0}$ and $n_{\nu}^{0}$ we have

$$
\max \prod_{i=1}^{p} n_{i}=n_{\mu}^{0} n_{\nu}^{0} \prod_{i \neq \mu, \nu} n_{i}^{0}=K n_{\mu}^{0} n_{\nu}^{0} .
$$

Here $K=\prod_{i \neq \mu, \nu} n_{i}^{0}$ is a certain constant, so that, from the result of $p=2, n_{\mu}^{0}$ and $n_{\nu}^{0}$ must satisfy that

$$
\left|n_{\mu}^{0}-n_{\nu}^{0}\right| \leqq 1
$$

This inequality should be constantly satisfied for all of pairs $\left(n_{\mu}^{0}, n_{\nu}^{0}\right)$ in $n_{1}^{0}, n_{2}^{0}, \cdots, n_{p}^{0}$. This completes the proof.

By using the result of De La Garza [5], Hoel [9] has derivated the result that the inverse of the generalized variance of the best linear estimators of coefficients $c_{0}, c_{1}, \cdots, c_{p-1}$ is given by

$$
\frac{1}{G . V .}=\frac{1}{\sigma^{2 p}} \prod_{i<j}^{p}\left(x_{i}-x_{j}\right)^{2} \prod_{i=1}^{p} n_{i} .
$$

The minimization of $G . V$. is equivalent to the maximization of $\prod_{i<j}\left(x_{i}-x_{j}\right)^{2}$ and that of $\prod_{i=1}^{p} n_{i}$ under the restriction $\sum_{i=1}^{p} n_{i}=$ constant. In this case, Schur [16] has proved that if $p \geqq 2$ and $-1 \leqq x_{i} \leqq 1(i=1,2, \cdots, p)$, then the zeros of integral of Legendre's polynomial maximize $\prod_{i<j}\left(x_{i}-x_{j}\right)^{2}$. We obtain then the following Theorem 1 by using these results and the prescribed lemma.

THEOREM 1. D-optimum design consists of such p supporting points as the zeros of $\int_{x}^{1} P_{p-1}(x) d x$, where " $a+1$ " observation is assigned in each of $\zeta$ supporting points 
and " a" observation is assigned in each of the $p-\zeta$ remainders respectively.

Hoel's result [9] is the special case of Theorem 1 for $\zeta=0$. Equation (3.3) for $D$-optimum design is given by

$$
\max _{d=\boldsymbol{\Delta}} \frac{1}{G \cdot V}=\frac{1}{\sigma^{2 p}} \frac{(p-1)^{p-1} \prod_{m=2}^{p-2} m^{2 m}}{\prod_{m=2}^{p-1}(2 m-1)^{2 m-1}}(n+p-\zeta) \cdot(n-\zeta)^{p-\zeta} .
$$

From Theorem 1, it follows that there are $\left(\begin{array}{c}p \\ \zeta\end{array}\right) D$-optimum designs which the $p$ supporting points are symmetric with respect to the original point for any $\zeta$.

In the proof of the theorem for $G(0, \sigma)$-optimum design, Guest [8] has shown that

$$
F^{\prime \prime}(x ; d)=0
$$

is the minimax condition. However, it is obvious that the condition (3.5) is not satisfied when $0<\zeta<p$. Therefore, in this case, the supporting points differ from the zeros of the integral of the Legendre's polynomial. By summarizing these results, we have:

$$
d^{*}(0, \sigma) \text { is equivalent to } D \text {-optimum design if and only if } \zeta=0 .
$$

Moreover the relationship between $G(0, \sigma), D$ and $I(0, \sigma, x)$-optimum designs is given by the following theorem.

TheOREM 2. When $n_{i}=a(i=1,2, \cdots, p), I(0, \sigma, x)$-optimum design, that is $d^{*}(0, \sigma, x)$, coincides with $d^{*}(0, \sigma)$ and D-optimum design.

Proof. On the basis of the definition of $I(0, \sigma, x)$-optimality, it is required that

$$
\sum_{i=1}^{p} \int_{-1}^{1} \frac{\partial L_{i}(x)}{\partial x_{k}} L_{i}(x) d x=0, \quad(k=1,2, \cdots, p) .
$$

According to (1.2), we have

Putting

$$
\frac{\partial}{\partial x_{k}} L_{i}(x)= \begin{cases}\left(\frac{1}{x_{i}-x_{k}}-\frac{1}{x-x_{k}}\right) L_{i}(x), & \text { for } k \neq i, \\ \left(\sum_{j}^{\prime} \frac{1}{x_{k}-x_{j}}\right) L_{i}(x), & \text { for } k=i .\end{cases}
$$

we have the relations

$$
f_{i}(x)=F(x) /\left(x-x_{i}\right),
$$

and

$$
F^{\prime}\left(x_{i}\right)=f_{i}\left(x_{i}\right)
$$

Now we have

$$
F^{\prime \prime}\left(x_{i}\right)=2 f_{i}^{\prime}\left(x_{i}\right) \text {. }
$$

$$
\sum_{i=1}^{p} \frac{1}{x-x_{i}}-\frac{1}{x-x_{k}}=\frac{f_{k}^{\prime}(x)}{f_{k}(x)}
$$

and then obtain

$$
\sum_{i}^{\prime} \frac{1}{x_{k}-x_{i}}=\frac{f_{k}^{\prime}\left(x_{k}\right)}{f_{k}\left(x_{k}\right)} \quad \text { for } \quad i \neq k .
$$

From these results, the requirement (3.6) is reduced to 


$$
\sum_{k=1}^{p} \frac{f_{k}^{\prime}\left(x_{k}\right)}{f_{k}\left(x_{k}\right)} \int_{-1}^{1} L_{k}^{2}(x) d x-\sum_{k=1}^{p} \int_{-1}^{1} \frac{f_{k}^{\prime}(x)}{f_{k}(x)} L_{k}^{2}(x) d x=0 .
$$

In the second term of the left hand side in this equation, we can obtain that

$$
\int_{-1}^{1} \frac{f_{k}^{\prime}(x)}{f_{k}(x)} L_{k}^{2}(x) d x=\frac{1}{f_{k}^{2}\left(x_{k}\right)} \int_{-1}^{1} f_{k}(x) f_{k}^{\prime}(x) d x=\frac{1}{2 f_{k}^{2}\left(x_{k}\right)}\left[f_{k}^{2}(x)\right]_{-1}^{1} .
$$

Hence the $p$ supporting points satisfied (3.6) are \pm 1 and $p-2$ roots of

$$
f_{k}^{\prime}\left(x_{k}\right)=\frac{1}{2}-F^{\prime \prime}\left(x_{k}\right)=0 \text {. }
$$

Schur [16] has shown that these $p$ points are the zeros of

$$
\int_{x}^{1} P_{p-1}(x) d x=\frac{1}{p(p-1)}\left(1-x^{2}\right) P_{p-1}^{\prime}(x) .
$$

This completes the proof.

When $0<\zeta<p$, the supporting points in $d^{*}(0, \sigma, x)$ are not always symmetric with respect to the original point. For example, when $p=2$, we obtain

$$
V[d, 0, \sigma, x]=\frac{2 \sigma^{2}}{\left(x_{1}-x_{2}\right)^{2}}\left[\frac{1}{n_{1}}\left(\frac{1}{3}+x_{2}^{2}\right)+\frac{1}{n_{2}}\left(\frac{1}{3}+x_{1}^{2}\right)\right] .
$$

Therefore the necessary condition for which a desigh $d \in \Delta$ is $I(0, \sigma, x)$-optimum is given by

$$
\frac{n_{1}^{2}}{n_{2}^{2}}=\frac{1+3 x_{2}^{2}}{1+3 x_{1}^{2}},
$$

so that if $\zeta \neq 0$ or $n_{1} \neq n_{2}$, then $x_{1}^{2} \neq x_{2}^{2}$ and thus $d^{*}(0, \sigma, x)$ is not symmetric and not coincides with $D$-optimum design.

In this section, it is assumed that any design $d_{0} \in J$ always satisfies the condition $E[\rho(x ; d)]=0$. Therefore if

$$
\left.\begin{array}{rlrl}
f^{(p)}(x) & \neq 0 & \text { for } & p<p_{0} \\
\equiv 0 & \text { for } & p \geqq p_{0}
\end{array}\right\}
$$

are assumed, then $E[\rho(x ; d)]=0$ is always satisfied by taking the $p$ supporting points more than $p_{0}$. When the value $p_{0}$ satisfying (3.9) exists exactly, we will study how far the change of value $p$ will affect $V\left[d^{*}(0, \sigma, x), 0, \sigma, x\right]$ and $V\left[d^{*}(0, \sigma), 0, \sigma\right]$.

In the seeking of $\hat{f}_{q}(x ; d)$, De La Garza [5] has shown that just as much information is obtained from observations made at certain $q+1$ supporting points in the interior of an interval as from $n(\geqq q+1 \geqq p)$ distinct points in that interval. Therefore it is sufficient to consider only $q+1$ supporting points for the seeking of an optimum design. Hence we will use Lagrange's interpolation formula for obtaining of $\hat{f}_{q}(x ; d)$. And if we put $q+1=p$, and $\zeta=0$, then, by using Guest's results [8], we have

$$
\sigma^{2} \sum_{i=1}^{p} \frac{1}{n_{i}} L_{i}^{2}\left(x ; d^{*}(0, \sigma)\right)=\frac{p \sigma^{2}}{n}\left[1+\frac{1}{p(p-1)}\left(x^{2}-1\right)\left\{P_{p-1}^{\prime}(x)\right\}^{2}\right] .
$$

Consequently the maximum expected squared error is given by

$$
V\left[d^{*}(0, \sigma), 0, \sigma\right]=p \sigma^{2} / n \text {. }
$$


When $n$ and $p$ were given, this value increases linearly as $p$ increases.

Now write

$$
F_{p}=\int_{x}^{1} P_{p-1}(x) d x=-\frac{1}{2^{p-2}}(p-1) ! \frac{d^{p-1}}{d x^{p-1}}\left(x^{2}-1\right)^{p-1},
$$

so that we obtain

$$
\begin{aligned}
\int_{-1}^{1}\left(x^{2}-1\right)\left\{P_{p^{-1}}^{\prime}(x)\right\}^{2} d x & =\frac{p}{2^{p-1}(p-2) !} \int_{-1}^{1} \frac{d^{p}\left(x^{2}-1\right)^{p-1}}{d x^{p}} F_{p} d x \\
& =(-1)^{p} \frac{p}{2^{p-1}(p-2) !} \int_{-1}^{1}\left(x^{2}-1\right)^{p-1} \frac{d^{p}}{d x^{p}} F_{p} d x .
\end{aligned}
$$

But we have

$$
\frac{d^{p}}{d x^{p}} F_{p}=-\frac{1}{2^{p-1}(p-1) !} \frac{d^{2 p-2}}{d x^{2 p-2}}\left\{x^{2 p-2}-(p-1) x^{2 p-4}+\cdots\right\}=-\frac{(2 p-2) !}{2^{p-1}(p-1) !} .
$$

and

$$
\int_{-1}^{1}\left(x^{2}-1\right)^{p-1} d x=(-1)^{p-1} \frac{2^{p}(p-1) !}{(2 p-1) ! !} \text {. }
$$

Hence, from (3.10) and the above results, we obtain

$$
V\left[d^{*}(0, \sigma, x), 0, \sigma, x\right]=\frac{2 p \sigma^{2}}{n}\left(1-\frac{1}{2 p-1}\right) .
$$

Consequently, when $n$ and $\sigma^{2}$ were given, the expected mean square error (3.12) increases as $p$ increases. We summarized our results in

THEOREM 3. If $\zeta=0$ and (3.9) hold, then the optimum number of the supporting points on $d^{*}(0, \sigma)$ and $d^{*}(0, \sigma, x)$ is exactly $p_{0}$.

Hoel [9] showed that the similar result is satisfied for $D$-optimum design by using the result of De La Garza [5].

\section{§4. Stochastic optimum design.}

In the unbiased stochastic case, it is easily shown that $I(0, \sigma, x)$ and $G(0, \sigma)$-optimum designs are optimum for all values of $\sigma^{2}$. Similarly $I\left(c_{p}, 0, x\right)$ and $G\left(c_{p}, 0\right)$-optimum designs are optimum for all values of $c_{p}$ in the deterministic case. However the optimum designs in the stochastic case depend upon the value of $\sigma /\left|f^{(p)}(\xi)\right|$ and then we will examine the change of such situation in some examples.

EXAMPLE 1. When

and

$$
p=2, \quad n_{1}=n_{2}=n / 2, \quad-x_{1}=x_{2}
$$

$$
f(x)=c_{0}^{\prime}+c_{1}^{\prime} P_{1}(x)+c_{2}^{\prime} P_{2}(x),
$$

David and Arens [4] investigated $d^{*}\left(3 c_{2}^{\prime}, \sigma, x\right)$ and $d^{*}\left(3 c_{2}^{\prime}, \sigma\right)$ in the specified values of $\sigma /\left|c_{2}^{\prime}\right|$. In this case, according to (1.5), we obtain

$$
E\left[\rho^{2}(x ; d, f)\right]=\left\{\frac{3}{2} c_{2}^{\prime}\left(x-x_{1}\right)\left(x-x_{2}\right)\right\}^{2}+\frac{2 \sigma^{2}}{n}\left\{\frac{\left(x-x_{1}\right)^{2}+\left(x-x_{2}\right)^{2}}{\left(x_{1}-x_{2}\right)^{2}}\right\} .
$$

Box and Draper [1] showed that the same result with David and Arens' $d^{*}\left(3 c_{2}^{\prime}, \sigma, x\right)$ is directly obtained by making use of our formula (4.1). Similarly, we can easily obtain $d^{*}\left(3 c_{2}^{\prime}, \sigma\right)$ from (4.1). 
Example 2. When

and

$$
p=3, \quad n_{i}=n / 3 \quad(i=1,2,3)
$$

$$
f(x)=\sum_{i=0}^{3} \frac{c_{i}}{i !} x^{i}
$$

we will examine the behaviours of $d^{*}\left(c_{3}, \sigma\right)$ and $d^{*}\left(c_{3}, \sigma, x\right)$ as functions of $\sigma^{2}$ and $c_{3}$.

If $J$ is restricted to the symmetric class of designs, i. e. $x_{1}=-x_{3} \neq 0, x_{2}=0$, $n_{i}=n / 3 \equiv n^{\prime}$, then we have

$$
E\left[\rho^{2}(x ; d, f)\right]=\frac{c_{3}^{2}}{36^{-}} x^{2}\left(x^{2}-x_{1}^{2}\right)^{2}+\frac{\sigma^{2}}{2 n^{\prime}}\left(3 \frac{x^{4}}{x_{1}^{4}}-3 \frac{x^{2}}{x_{1}^{2}}+2\right) .
$$

We put $A_{1}=c_{3}^{2} / 36, \quad A_{2}=\sigma^{2} / 2 n^{\prime}, y=x^{2}$ and $\gamma=x_{1}^{2}$ in (4.2). Hence it is required to maximize

$$
P=\left[A_{1} y^{2}+\left(-2 A_{1} \gamma+\frac{3 A_{2}}{\gamma^{2}}\right) y+A_{1} \gamma^{2}-\frac{3 A_{2}}{\gamma}\right] y
$$

with respect to $y \in[0,1]$ and subsequently to minimize this maximum with respect to $\gamma \in[0,1]$. The roots of $P$ are $0, \gamma$ and $\gamma-\frac{3 A_{2}}{A_{1} \gamma^{2}}$. Therefore when $0<\gamma^{3} \leqq \frac{3 A_{2}}{A_{1}}$, the maximum of $P$ occurs at $y=1$. Now

$$
P(y=1)=A_{1}(1-\gamma)^{2}+\frac{3 A_{2}}{\gamma^{2}}(1-\gamma)>0
$$

and this value decreases in $\gamma$ from $+\infty$ to 0 . Thus if $3 A_{2} / A_{1} \geqq 1$, then $\gamma=1$ is the solution. Otherwise if $0 \leqq \frac{3 A_{2}}{A_{1}}<\gamma^{3}$, then the maximum of $P$ occurs at $y=1$ or the smallest root of $\frac{\partial P}{\partial \gamma}=0$;

$$
y_{\gamma}=\frac{1}{3 A_{1}}\left(2 A_{1} \gamma-\frac{3 A_{2}}{\gamma^{2}}-\sqrt{A_{1}^{2} \gamma^{2}-\frac{3 A_{1} A_{2}}{\gamma}+\frac{9 A_{2}^{2}}{\gamma^{4}}}\right) .
$$

However $\frac{\partial}{\partial x} P\left(y=y_{r}\right)>0$, so that $P\left(y=y_{r}\right)$ increases in $\gamma\left(>\left(\frac{3 A_{2}}{A_{1}}\right)^{1 / 3} \geqq 0\right)$ from 0 to $P\left(y=y_{1}\right)$. Therefore the solution in this case is the root of the equation $P\left(y=y_{\gamma}\right)$ $=P(y=1)$. Hence we must calculate this solution $\gamma_{0}\left(>\left(\frac{3 A_{2}}{A_{1}}\right)^{1 / 3} \geqq 0\right)$ for the given values of $A_{1}$ and $A_{2}$. Specially, when $A_{2}=0$, according to Guest's result for $G\left(c_{p}, 0\right)$. optimum design in section 3 , we obtain $\gamma_{0}=3 / 4$. We summarized our results: for $p=3$, the symmetric $G\left(c_{3}, \sigma\right)$-optimum design is obtained as follows.

(i) If $\frac{\sigma}{\left|c_{3}\right|} \geqq \frac{\sqrt{n^{\prime}}}{3 \sqrt{6}}$ then the supporting points are the zeros of Legendre's integral $\int_{x}^{1} P_{2}(x) d x$, i. e., $x_{1}=-1, x_{2}=0, x_{3}=1$.

(ii) If $\frac{\sqrt{n^{\prime}}}{3 \sqrt{ } 6}>\frac{\sigma}{\left|c_{3}\right|}$ then we must calculate the solution by the numerical method as is stated above.

(iii) If $\sigma=0$, then the supporting points are the zeros of Chebyshev's polynomial $T_{s}(x)$, i. e., $x_{1}=-\sqrt{3} / 2, x_{2}=0, x_{3}=\sqrt{3} / 2$.

On the other hand, from (4.2) we have 


$$
V\left[d, c_{3}, \sigma, x\right]=\frac{c_{3}^{2}}{18}\left(\frac{1}{7}-\frac{2}{5} x_{1}^{2}+\frac{1}{3}-x_{1}^{4}\right)+\frac{\sigma^{2}}{n^{\prime} x_{1}^{4}}\left(\frac{3}{5}-x_{1}^{2}+2 x_{1}^{4}\right) \equiv B+V \quad \text { (say) } .
$$

The value of $V\left[d, c_{3}, \sigma, x\right]$ is convex in $x_{1}^{2} \subseteq(0,1]$ and then $d^{*}\left(c_{3}, \sigma, x\right)$ is unique for given $\sigma /\left|c_{3}\right|$. When $0<x_{1}^{2} \leqq 3 / 5, B$ and $V$ decrease as $x_{1}^{2}$ increases respectively. Otherwise when $3 / 5 \leqq x_{1}^{2} \leqq 1, B$ increases and $V$ decreases as $x_{1}^{2}$ increases. Hence $V\left[d, c_{3}, \sigma, x\right]$ is uniquely minimized at $x_{1}^{2} \in\left[\frac{3}{5}, 1\right]$ and its minimized value $x_{1}^{2}$ depends upon $\sigma /\left|c_{3}\right|$.

Specially, if $c_{3}=0$, then the solution is $x_{1}^{2}=1$ and if $\sigma=0$, then the solution is $x_{1}^{2}=3 / 5$. Hence, for $p=3$, the symmetric $I\left(c_{3}, \sigma, x\right)$-optimum design is obtained as follows.

(i) If $\frac{\sigma}{\left|c_{3}\right|} \geqq \frac{2 \sqrt{n^{\prime}}}{27}$, then the supporting points are the zeros of Legendre's integral $\int_{x}^{1} P_{2}(x) d x$, i. e., $x_{1}=-1, x_{2}=0, x_{3}=1$.

(ii) If $\frac{2 \sqrt{n^{\prime}}}{27}>\frac{\sigma}{\left|c_{3}\right|}>0$, then the solution is

$$
x_{1}=-\sqrt{\gamma_{0}}, \quad x_{2}=0, \quad x_{3}=\sqrt{\gamma_{0}},
$$

where $\gamma_{0}$ is the root of the equation

$$
5 \gamma^{4}-3 \gamma^{3}+27 \frac{\sigma^{2}}{n^{\prime} c_{3}^{2}}(5 \gamma-6)=0 \text { and } \gamma_{0} \in\left(\frac{3}{5}, 1\right) .
$$

(iii) If $\sigma=0$, then the supporting points are the zeros of Legendre polynomial $P_{3}(x)$, i. e., $x_{1}=-\sqrt{ } 3 / 5, x_{2}=0, x_{3}=\sqrt{3 / 5}$.

So far for our two examples. In general, it is, however, difficult to determine exactly the optimum designs in stochastic case for $p \geqq 4$.

Moreover even if they are determined, the functional form of $f(x)$ and $\sigma^{2}$ are often unknown or only approximately known at the beginning of the experiment. We also pointed out in this section that an optimum design in stochastic case depends upon the value of $\sigma /\left|f^{(p)}(\xi)\right|$. Hence, we consider the effect of this value to the efficiencies defined in section 1 for the designs $d^{*}\left(c_{p}, 0\right), d^{*}(0, \sigma), d^{*}\left(c_{p}, 0, x\right)$ and $d^{*}(0, \sigma, x)$ which are optimum under the assumption $\sigma=0$ or $\left|f^{(p)}(\xi)\right|=0$ in section 2 and 3 respectively. We shall call these designs as extreme designs. These are equivalent to examine the change of efficiencies of the extreme designs according to the change of $\sigma /\left|f^{(p)}(\xi)\right|$. Namely, if we use the extreme designs, in the case when the information related to $\sigma /\left|f^{(p)}(\xi)\right|$ is not known at all, then the question which would naturally arise would be "At what value of $\sigma /\left|f^{(p)}(\xi)\right|$ would each of these extreme (optimum) designs attain its minimum efficiencies?".

To begin with, we will examine in detail the change of the efficiencies of extreme designs for $p=2$ and 3 in this section. The efficiencies of the extreme designs: $d^{*}\left(c_{2}, 0, x\right), d^{*}(0, \sigma, x), d^{*}\left(c_{2}, 0\right)$ and $d^{*}(0, \sigma)$ are given in Table I on the basis of $I\left(c_{2}, \sigma, x\right)$ and $G\left(c_{2}, \sigma\right)$-optimum designs against the given values of $\sigma /\left|c_{2}\right|$ which have been studied by David and Arens [4]. And $I\left(c_{3}, \sigma, x\right)$ and $G\left(c_{3}, \sigma\right)$-optimum designs and the efficiencies of the extreme designs $d^{*}\left(c_{3}, 0, x\right), d^{*}(0, \sigma, x), d^{*}\left(c_{3}, 0\right)$ and $d^{*}(0, \sigma)$ are given. in Table II. Here $c_{2}=3 c_{2}^{\prime}$. 
TABLE I

Symmetric $I\left(c_{2}, \sigma, x\right)$ and $G\left(c_{2}, \sigma\right)$-optimum designs and efficiencies of extreme designs.

\begin{tabular}{|c|c|c|c|c|c|c|}
\hline \multirow{2}{*}{$\sqrt{\frac{2}{n}} \frac{\sigma}{\left|c_{2}{ }^{\prime}\right|}$} & \multicolumn{2}{|c|}{$-x_{1}=x_{2}$} & \multirow{2}{*}{$\begin{array}{r}e\left[d^{*}(0, \sigma, x)\right. \\
\left.\mid c_{2}, \sigma, x\right]\end{array}$} & \multirow{2}{*}{$\begin{array}{r}e^{-} d^{*}\left(c_{2}, 0, x\right) \\
\mid c_{2}, \sigma, x\end{array}$} & \multirow{2}{*}{$\begin{array}{r}e_{-}^{-} d^{*}\left(c_{2}, 0\right) \\
\left.\mid c_{2}, \sigma\right\rfloor\end{array}$} & \multirow{2}{*}{$\begin{array}{r}e\left[d^{*}(0, \sigma)\right. \\
\left.\mid c_{2}, \sigma\right]\end{array}$} \\
\hline & $d^{*}\left(c_{2}, \sigma, x\right)$ & $d^{*}\left(c_{2}, \sigma\right)$ & & & & \\
\hline 0 & 0.577 & 0.707 & 0.167 & 1.000 & 1. 000 & 0.250 \\
\hline 0.2 & 0.642 & 0.755 & 0.375 & 0.963 & 0.827 & 0.374 \\
\hline 0.4 & 0.725 & 0.850 & 0.676 & 0.890 & 0.695 & 0.636 \\
\hline 0.6 & 0.796 & 0.949 & 0.856 & 0.835 & 0.635 & 0.889 \\
\hline 0.8 & 0.855 & 1.000 & 0.942 & 0.796 & 0.626 & 1.000 \\
\hline 1.0 & 0.908 & - & 0.981 & 0.767 & 0.640 & - \\
\hline 1.2 & 0.955 & - & 0.966 & 0.745 & & - \\
\hline 1.4 & 0.997 & - & 1.000 & 0.726 & & - \\
\hline 1.6 & 1.000 & - & - & 0.713 & & - \\
\hline$\infty$ & 1.000 & 1.000 & 1.000 & 0.667 & 0.667 & 1.000 \\
\hline
\end{tabular}

In this example $(p=2)$, we obtain the minimum efficiencies of each extreme designs as follows.

$$
\begin{array}{ll}
\min _{\sigma /\left|c_{2}\right|} e\left[d^{*}\left(c_{2}, 0, x\right) \mid c_{2}, \sigma, x\right]=2 / 3 & \text { for } \quad c_{2}=0 \\
\min _{\sigma /\left|c_{2}\right|} e\left[d^{*}(0, \sigma, x) \mid c_{2}, \sigma, x\right]=1 / 6 & \text { for } \sigma=0 \\
\min _{\sigma /\left|c_{2}\right|} e\left[d^{*}\left(c_{2}, 0\right) \mid c_{2}, \sigma\right]=7 / 13 & \text { for } \frac{2}{n}\left(\begin{array}{c}
\sigma \\
c_{2}^{\prime}
\end{array}\right)^{2}=\frac{1}{2} \\
\min _{\sigma /\left|c_{2}\right|} e\left[d^{*}(0, \sigma) \mid c_{2}, \sigma\right]=1 / 4 & \text { for } \sigma=0 .
\end{array}
$$

Then we know that the lowest efficiency of $d^{*}\left(c_{2}, 0\right)$ (and $d^{*}\left(c_{2}, 0, x\right)$ ) is better than

\begin{tabular}{|c|c|c|c|c|c|c|}
\hline \multirow{2}{*}{$\frac{\sigma}{\sqrt{n}\left|c_{3}\right|}$} & \multicolumn{2}{|c|}{$-x_{1}=x_{2}$} & \multirow{2}{*}{$\begin{array}{r}e\left[d^{*}\left(c_{3}, 0, x\right)\right. \\
\left.\mid c_{3}, \sigma, x\right]\end{array}$} & \multirow{2}{*}{$\begin{array}{r}e\left[d^{*}(0, \sigma, x)\right. \\
\left.\mid c_{3}, \sigma, x\right]\end{array}$} & \multirow{2}{*}{$\begin{array}{r}e_{[}^{-} d^{*}\left(c_{3}, 0\right) \\
\left.\mid c_{3}, \sigma\right]\end{array}$} & \multirow{2}{*}{$\begin{array}{r}e\left[d^{*}(0, \sigma)\right. \\
\left.\mid c_{3}, \sigma\right]\end{array}$} \\
\hline & $d^{*}\left(c_{3}, \sigma, x\right)$ & $d^{*}\left(c_{3}, \sigma\right)$ & & & & \\
\hline 0 & 0.7746 & 0.8660 & 1.000 & 0.300 & 1.000 & 0.482 \\
\hline 0.0504 & 0.8367 & & 0.948 & 0.725 & & \\
\hline 0.0974 & 0.8944 & & 0.890 & 0.928 & & \\
\hline 0.1361 & & 1.0000 & & & 0.568 & 1.000 \\
\hline 0.1863 & 0.9487 & - & 0.842 & 0.975 & 0.583 & - \\
\hline 0.2722 & 1.000 & - & 0.822 & 1.000 & 0.592 & - \\
\hline- & - & - & & - & & - \\
\hline$\infty$ & 1. 0000 & 1.0000 & 0.800 & 1.000 & 0.600 & 1.000 \\
\hline
\end{tabular}
that of $d^{*}(0, \sigma)$ (and $\left.d^{*}(0, \sigma, x)\right)$.

TABLE II

Symmetric $I\left(c_{3}, \sigma, x\right)$ and $G\left(c_{3}, \sigma\right)$-optimum designs and efficiencies of extreme designs. 


\section{§. Efficiencies of extreme designs.}

When the nature of $f(x)$ and $\sigma^{2}$ are unknown, $d^{*}\left(c_{p}, 0, x\right), d^{*}(0, \sigma, x), d^{*}\left(c_{p}, 0\right)$ and $d *(0, \sigma)$ will be called the extreme designs as was mentioned in section 4 . For an arbitrary $p$ we examine such lowest efficiencies of the extreme designs as we have given in (4.3) for $p=2$. In order to do this, we will first give the expected mean square error and the maximum expected square error for these designs respectively.

(i) From Theorem 2 for $I\left(c_{p}, 0, \alpha\right)$-optimum design, we have

$$
V\left[d^{*}\left(c_{p}, 0, \alpha\right), f^{(p)}, \sigma, \alpha\right]=\left\{\frac{f^{(p)}(\xi)}{p ! \beta_{p}}\right\}^{2} \int_{-1}^{1} \phi_{p}(x) d \alpha(x)+\sigma^{2} \sum_{i=1}^{p} \frac{\lambda_{i}}{n_{i}},
$$

where $\beta_{p}$ is a coefficient in $x^{p}$ of $\phi_{p}(x)$ and $\lambda$ 's are Christoffel numbers

$$
\lambda_{i}=\int_{-1}^{1}\left\{\frac{\phi(x)}{\left(x-x_{i}\right) \phi_{p}^{\prime}\left(x_{i}\right)}\right\}^{2} d \alpha(x), \quad(i=1,2, \cdots, p) .
$$

The first term of the right hand side of (5.1) is obtained from the second law of the mean $[10]$ and

$$
F\left(x ; d^{*}\left(c_{p}, 0, \alpha\right)\right)=\dot{\phi}_{p}(x) / \beta_{p} .
$$

Since the property of $d *\left(c_{p}, 0, \alpha\right)$ satisfies the condition of Theorem 14.2.1 by Szegö [18],

$$
\int_{-1}^{1} L_{i}\left(x ; d^{*}\left(c_{p}, 0, \alpha\right)\right) L_{j}\left(x ; d^{*}\left(c_{p}, 0, \alpha\right)\right) d \alpha(x)=\lambda_{i} \delta_{i j}, \quad(i, j=1,2, \cdots, p)
$$

holds. Hence we obtain the second term of the right hand side of (5.1). When $n_{i}=n / p$, we have

$$
V\left[d^{*}\left(c_{p}, 0, \alpha\right), f^{(p)}, \sigma, \alpha\right]=\left\{\frac{f^{(p)}(\xi)}{p ! \beta_{p}}\right\}^{2} \int_{-1}^{1} \phi_{p}(x) d \alpha(x)+\frac{p \sigma^{2}}{n}\{\alpha(1)-\alpha(-1)\},
$$

by using Theorem 3.4.2 by Szegö [18]. And from $\phi_{p}(x)=P_{p}(x)$ for $d^{*}\left(c_{p}, 0, x\right)$, it follows that

$$
V\left[d^{*}\left(c_{p}, 0, x\right), f^{(p)}, \sigma, x\right]=\frac{2^{2 p+1}(p !)^{2}}{(2 p+1)\{(2 p) !\}^{2}} \cdot\left\{f^{(p)}(\xi)\right\}^{2}+\sigma^{2} \sum_{i=1}^{p} \frac{\lambda_{i}}{n_{i}} .
$$

Here if $n_{i}=n / p(i=1,2, \cdots, p)$, then we have

$$
\sum_{i=1}^{p} \lambda_{i} / n_{i}=2 p / n
$$

Similarly, $\phi_{p}(x)=T_{p}(x)$ holds for $d^{*}\left(c_{p}, 0,\left(1-x^{2}\right)^{-\frac{1}{2}}\right.$ ), (where $T_{p}(x)$ is Chebyshev's polynomial of degree $p$ ), so that it follows that

$$
V\left[d^{*}\left(c_{p}, 0,\left(1-x^{2}\right)^{-\frac{1}{2}}\right), f^{(p)}, \sigma,\left(1-x^{2}\right)^{-\frac{1}{2}}\right]=\frac{\pi}{2^{2 p-1}(p !)^{2}}\left\{f^{(p)}(\xi)\right\}^{2}+\sigma^{2} \sum_{i=1}^{p} \lambda_{i} .
$$

Here if $n_{i}=n / p(i=1,2, \cdots, p)$, then we have

$$
\sum_{i=1}^{p} \lambda_{i} / n_{i}=\pi p / n
$$

For $d^{*}\left(c_{p}, 0\right)$ we obtain

$$
V\left[d^{*}\left(c_{p}, 0\right), f^{(p)}, \sigma\right]=\max _{-1 \leqq x \leqq 1}\left[\left\{\frac{T_{p}(x)}{2^{p-1} p !} f^{(p)}(\xi)\right\}^{2}+\sigma^{2} \sum_{i=1}^{p} \frac{1}{n_{i}}\left\{\frac{T_{p}(x)}{\left(x-x_{i}^{0}\right)^{2} T_{p}^{\prime}\left(x_{i}^{0}\right)}\right\}\right],
$$

where $x_{i}^{0}=\cos [(2 i-1) / 2 p](i=1,2, \cdots, p)$ are the zeros of $T_{p}(x)$. Specially, when 
$\sigma^{2}=0$,

holds.

$$
V\left[d *\left(c_{p}, 0\right), c_{p}, 0\right]=\left(\frac{c_{p}}{2^{p-1} p !}\right)^{2}
$$

(ii) From Theorem 2 for $I(0, \sigma, x)$-optimum design, we find

$$
\begin{aligned}
F(x ; d *(0, \sigma, x)) & =K\left(x^{2}-1\right) P_{p-1}^{\prime}(x) \\
& =K\left(x^{2}-1\right) 2^{1} 2^{p-1} \sum_{j=0}^{\leqq(p-2) / 2} j !(p-j-1) !(p-2 j-2) ! x^{p-2 j-2} .
\end{aligned}
$$

Here the coefficient of $x^{p}$ must be equal to one, so that we have

We obtain therefore

$$
K=2^{p-1}(p-1) !(p-2) ! /(2 p-2) ! .
$$

$$
E^{2}\left[\rho\left(x ; d^{*}(0, \sigma, x)\right)\right]=\left\{\frac{2^{p-1}(p-2) !}{p(2 p-2) !}\left(x^{2}-1\right) P_{p-1}^{\prime}(x) f^{(p)}(\xi)\right\}^{2} .
$$

By making use of the similar method to give (3.12), we can show that

$$
\int_{-1}^{1}\left(x^{2}-1\right)^{2}\left\{P_{p-1}^{\prime}(x)\right\}^{2} d x=\frac{\{2 p(p-1)\}^{2}}{(2 p-1)(2 p-1)(2 p-3)} .
$$

From these results and (3.12) we obtain

$$
\begin{aligned}
& V\left[d^{*}(0, \sigma, x), f^{(p)}, \sigma, x\right] \\
& \quad=\quad \frac{2^{p}(p-2) !}{(2 p-3)^{2}(2 p-2) !(2 p-1) ! !}\left\{f^{(p)}(\xi)\right\}^{2}+\frac{2 p \sigma^{2}}{n}\left(1-\frac{1}{2 p-1}\right) .
\end{aligned}
$$

And from (3.10) and (5.9) we obtain

$$
\begin{aligned}
V\left[d^{*}(0, \sigma), f^{(p)}, \sigma\right]= & \max _{-1 \leqq x \leqq 1}\left[\left\{\begin{array}{c}
2^{p-1}(p-2) ! \\
p(2 p-2) !
\end{array}\left(x^{2}-1\right) P_{p-1}^{\prime}(x) f^{(p)}(\xi)\right\}^{2}\right. \\
& \left.+\frac{p \sigma^{2}}{n}\left\{1+\frac{1}{p(p-1)}\left(x^{2}-1\right)\left(P_{p-1}^{\prime}(x)\right)^{2}\right\}\right] .
\end{aligned}
$$

(iii) Using the results in (5.3), (5.4) and (5.10), when $n_{i}=n / p(i=1,2, \cdots, p)$, the relation

$$
V\left[d, c_{p}, \sigma, x\right] \geqq \frac{2^{2 p+1}(p !)^{2}}{(2 p+1)\{(2 p) !\}^{-2}} c_{p}^{2}+\frac{2 p \sigma^{2}}{n}\left(1-\begin{array}{c}
1 \\
2 p-1
\end{array}\right) \equiv V_{0} \quad \text { (say) }
$$

holds for any design $d \in \Delta$.

(iv) Likewise from (1.5), (1.4) and (1.7) we have

$$
V\left[d, f^{(p)}, \sigma\right] \geqq \max _{-1 \leqq x \leqq 1}\left\{F(x) \frac{f^{(p)}(\xi)}{p !}\right\}^{2},
$$

and

$$
V\left[d, f^{(p)}, \sigma\right] \geqq \sigma^{2} \max _{-1 \leqq x \leqq 1}\left[\sum_{i=1}^{p} \frac{1}{n_{i}} L_{i}^{2}(x)\right] .
$$

Moreover from (3.11) and (5.8) we obtain

$$
V\left[d, c_{p}, \sigma\right] \geqq\left(\frac{c_{p}}{2^{p-1} p !}\right)^{2},
$$

and

$$
V\left[d, c_{p}, \sigma\right] \geqq p \sigma^{2} / n
$$


Therefore the relation

$$
V\left[d, c_{p}, \sigma\right] \geqq \max \left[\left(\begin{array}{c}
c_{p} \\
2^{p-1} p !
\end{array}\right)^{2}, \begin{array}{c}
p \sigma^{2} \\
n
\end{array}\right] \equiv V_{0}^{\prime} \quad \text { (say) }
$$

holds for any design $d \in \mathcal{A}$.

Now let us turn to the calculations of efficiency of extreme designs in view of the results obtained in (i), (ii) and (iii). Indeed, the lower bounds of the efficiencies of $d^{*}\left(c_{p}, 0, x\right)$ and $d^{*}(0, \sigma, x)$ specified $n_{i}=n / p(i=1,2, \cdots, p)$ are given by

$$
e\left[d^{*}\left(c_{p}, 0, x\right) \mid c_{p}, \sigma, x\right] \geqq V_{0} /\left[(5.3) \text { specified }(5.4) \text { and } f^{(p)}(\hat{\xi})=c_{p}\right],
$$

and

$$
e\left[d^{*}(0, \sigma, x) \mid c_{p}, \sigma, x\right] \geqq V_{0} /\left[(5.10) \text { specified } f^{(p)}(\xi)=c_{p}\right]
$$

with strict inequality unless $c_{p}$ or $\sigma$ is zero. Hence the minimum efficiencies of these designs are given by

and

$$
\min _{\sigma /\left|c_{p}\right|} e\left[d^{*}\left(c_{p}, 0, x\right) \mid c_{p}, \sigma, x\right]=e\left[d^{*}\left(c_{p}, 0, x\right) \mid 0, \sigma, x\right]=1-\frac{1}{2 p-1},
$$

$$
\min _{\sigma /|c p|} e\left[d^{*}(0, \sigma, x) \mid c_{p}, \sigma, x\right]=e\left[d *(0, \sigma, x) \mid c_{p}, 0, x\right]=\frac{(2 p-3)}{2(2 p-1)} .
$$

We therefore obtain the following theorem.

THEOREM 4. When $n_{i}=n / p(i=1,2, \cdots, p)$, the minimum efiticiency on $d^{*}\left(c_{p}, 0, x\right)$ is better than that of $d^{*}(0, \sigma, x)$, that is

$$
\min _{\sigma / \mid c_{p} !} e\left[d^{*}\left(c_{p}, 0, x\right) \mid c_{p}, \sigma, x\right]=1-\frac{1}{2 p-1}>\min _{\sigma /\left|c_{p}\right|}\left[d^{*}(0, \sigma, x) \mid c_{p}, \sigma, x\right]=\frac{2 p-3}{2(2 p-1)} \cdot
$$
holds.

This theorem shows that if $c_{p}$ and $\sigma$ are unknown and if we consider the lowest efficiency of design, then $I\left(c_{p}, 0, x\right)$-optimum design in the numerical analysis is better than $I(0, \sigma, x)$-optimum design in the mathematical statistics.

Now let us turn to the application of the results obtained in (i), (ii) and (iv). When $n_{i}=n / p(i=1,2, \cdots, p)$, from (5.7) and (5.8) we have the relation

$$
\begin{aligned}
e\left[d^{*}\left(c_{p}, 0\right) \mid c_{p}, \sigma\right] & \geqq V_{0}^{\prime} /\left[(5.7) \text { specified } f^{(p)}(\xi)=c_{p} \text { and } n_{i}=n_{p}^{n}\right] \\
& >V_{0}^{\prime} /\left[\left(\frac{c_{p}}{2^{p-1} \bar{p} !}\right)^{2}+\frac{p \sigma^{2}}{n} \max _{-1 \leqq x \leqq 1}\left(\sum_{i=1}^{n}\left\{\frac{T_{p}(x)}{\left(x-x_{i}^{0}\right) T_{p}^{\prime}\left(x_{i}^{0}\right)}\right\}^{2}\right] .\right.
\end{aligned}
$$

When $\left(c_{p} / 2^{p-1} p !\right)^{2} \geqq p \sigma^{2} / n,\left(\left(c_{p} / 2^{p-1} p !\right)^{2} \leqq p \sigma^{2} / n\right)$, the last term in this inequality decreases (increases) as $\sigma /\left|c_{p}\right|$ increases. Hence we have

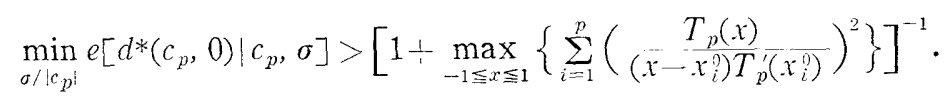

Similarly, from (5.11) and (3.11) we have the relation

$$
\begin{aligned}
e\left[d *(0, \sigma) \mid c_{p}, \sigma\right] & \geqq V_{0}^{\prime} /\left[(5.11) \text { specified } f^{(p)}(\xi)=c_{p}\right] \\
& >V_{0}^{\prime} /\left[\left\{\frac{2^{p-1}(p-2)}{p(2 p-2) !} c_{p}\right\}^{2} \max _{-1 \leqq x \leqq 1}\left\{\left(x^{2}-1\right) P_{p-1}^{\prime}(x)\right\}^{2}+\frac{p \sigma^{2}}{n}\right] .
\end{aligned}
$$

Therefore we obtain 


$$
\begin{aligned}
\min _{\sigma, j^{\prime}} e\left[d *(0, \sigma), c_{i}, \sigma\right] & >\left[1 \div\left\{\frac{2^{2 p-2}(p-1) !(p-2) !}{(2 p-2) !}\right\}^{2} \max \left\{\left(x^{2}-1\right) P_{p-1}^{\prime}(x)\right\}^{2}\right]^{-1} \\
& =\left[1 \div\left\{\begin{array}{c}
2^{p-2}(p-1) ! p ! \\
(2 p-2) !
\end{array}\right\}^{2}\left\{\int_{M}^{1} P_{p-1}(x) d x\right\}^{2}\right]^{-1}
\end{aligned}
$$

where $M=\min \left\{\left|x_{i}\right| P_{p-1}\left(x_{i}\right)=0\right\}$. Then we know that the equalities corresponding to $(5.16)$ and $(5.17)$ cannot are obtained in this case, but when $n_{i}=n / p(i=1,2, \cdots, p)$ we have

$$
e\left[d^{*}\left(c_{p}, 0\right) \mid 0, \sigma\right]=\left[\max _{-1 \leqq x \leqq 1}\left(\sum_{i=1}^{p}\left\{\frac{T_{p}(x)}{\left(x-\bar{x}(\overline{0}) \overline{T_{p}^{(}\left(x_{i}^{(}\right)}\right.}\right\}^{2}\right)\right]^{-1}
$$

and

$$
e\left[d *(0, \sigma) \mid c_{p}, 0\right]=\left\{\frac{(2 p-2) !}{2^{2 p-2} p !(p-1) !}\right\}^{2} /\left\{\int_{M}^{1} P_{p-1}(x) d x\right\}^{2}
$$

EXAMPLE 3. When $p=2, n_{1}=n_{2}=n_{i} / 2$ and $x_{1}=-x_{2}$,

$$
\left.\min _{\sigma / r_{2} \mid} e\left[d^{*}\left(c_{2}, 0\right) \mid c_{2}, \sigma\right)\right]=7 / 13, \quad \text { for }-\frac{2}{n}\left(\frac{\sigma}{c_{2}}\right)^{2}=\frac{1}{2},
$$

and

$$
e\left[d^{*}\left(c_{2}, 0\right) \mid 0, \sigma\right]=2 / 3
$$

hold, so that the equality corresponding to (5.16) is not satisfied. However the equality corresponding to $(5.17)$

ho!ds.

$$
\min _{\sigma / \mid c_{2}} e\left[d *(0, \sigma) \mid c_{2}, \sigma\right]=e\left[d *(0, \sigma) \mid c_{2}, 0\right]=1 / 4
$$

\section{References}

[1] G.E.P. Box and N.R. DRAPER, "A basis for the selection of a response surface design", J.R. Stat. Soc., 54 (1959), 622-654.

[2] G.E.P. BOX and N.R. DRAPER, "The choice of a second order rotatable design", Biometrica., 50 (1963), 335-352.

[3 H. Cherioff, "Locally optimum designs for estimating parameter", Ann. Math. Stat., 24 (1953), 586-602.

[4] H. A. DAvid and E. E. Arexs, "Optimal spacing in regression analysis", Ann. Math. Stat., 30 (1959), 1072-1081.

[5] A. De LA GARzA, "Spacing of information in polynomial regression", Ann. Math. Stat., 25 (1954), 123-130.

[6] S. Ehrejfeld, "On the efficiency of experimental designs", Ann. Math. Stat., 26 (1955), 247-255.

[7] G. Elfyisg, "Optimum allocation in linear regression theory", Ann. Math. Stat., 23 (1952), 255-262.

[8- P.G. GuEst, "The spacing of observations in polynomial regression", Ann. Math. Stat., 29 (1958), 294-299.

[9] P.G. HoEl, "Efficiency problems in polynomial estimation", Ann. Math. Stat., 29 (1958), $1134-1145$.

[10 F. B. Hildebraxd, Introduction to Numerical Analysis, McGrow-Hill, New York, (1956).

[11 J. Kieferand J. Wolfowitz, "Optimum design in regression problems", Ann. Math. Stat., 30 (1959), 271-294.

[12. J. Kiefer, „ "Optimum designs in regression problems, II", Ann. Math. Stat., 32 (1961), $298-325$. 
[13] J. Kiefer, “Optimum experimental designs”, J. R. Stat. Sec., Ser. B21, 272-319.

[14] T. KitAGAwA, "Some aspects of stochastically approximative analysis", Bull. Math. Stat., 6 (1956), 109-129.

[15] Z. KopAl, Numerical Analysis, Joen Wiley and Sons, New York, (1955).

[16] I. Schur, "Über die Verteilung der Wurgeln bei gewissen algebraischen Gleichung mit ganzzahligen Koeffizieten", Math. Zeit., (1918), 378.

[17] K. Sмiтн, "On the standard deviations of adjusted and interpolated values of an observed polynomial functiou and its constants and the guidance they give towards a proper choice of the distribution of observations", Biometrika, 12 (1918), 1-85.

[18] G. Szegö, Orthogonal polynomials, Ann. Math. Soc. Coll. Prob., 23, New York, (1939). 